\title{
Ictal Spitting in a Patient with Dominant Temporal Lobe Epilepsy: Supporting Evidence of Ictal Spitting from the Nondominant Hemisphere
}

\author{
Sea-Mi Park ${ }^{\mathrm{a}}$ Sang-Ahm Lee ${ }^{\mathrm{a}} \quad$ Ji Hyun Kim $^{\mathrm{b}}$ Joong Koo Kang ${ }^{\mathrm{a}}$ \\ ${ }^{a}$ Department of Neurology, Asan Medical Center, University of Ulsan College of Medicine, and \\ ${ }^{b}$ Department of Neurology, Guro Hospital, University of Korea College of Medicine, Seoul, South Korea
}

\section{Dear Sir,}

Automatisms are traditionally defined as stereotyped nonpurposeful automatic behaviors during a complex partial seizure, and the automatisms known to have a lateralizing value in temporal lobe epilepsy (TLE), spitting automatisms, though rarely reported, have been predominantly associated with nondominant temporal lobe lateralization. Although the pathophysiological mechanism of spitting automatism has not been fully defined, it has been hypothesized that the right hemispheric dominance of spitting automatism is due to the functional asymmetry of the central autonomic network [1], such as ictal vomiting or ictal urinary urge $[2,3]$. There have been rare reports of ictal spitting in dominant hemispheric seizures, but in such cases it has not been well explained whether the symptomatogenic ictal spitting area originates from the dominant hemisphere.

Here, we present the case of a righthanded TLE patient with ictal spitting, whose seizures originated from the left temporal lobe. An intracarotid amobarbital procedure (IAP) demonstrated left hemispheric language dominance. In the described case, the epileptogenic area proved to be in the left temporal lobe and the symptomatogenic area of ictal spitting was located to the right hemisphere, most likely the temporal lobe.
Case Report

A 28-year-old right-handed man with an 11-year history of intractable seizures underwent a comprehensive presurgical evaluation. His past history was unremarkable for febrile convulsion, head trauma, meningoencephalitis, or other primary neurologic diseases, and there was no family history of epilepsy. He first experienced a sudden loss of consciousness at age 17. Thereafter, a similar event occurred several times per month. He always felt the same indescribable unpleasant sensation, and could not recall any event. His family members noted that he looked blank, did not respond, fumbled with something using both hands, repetitively spat at the ground, and drooled. An antiepileptic drug (valproic acid 1,200 mg/ day) was prescribed after a diagnosis of epilepsy. However, despite the administrations of multiple drugs (valproic acid $1,200 \mathrm{mg} /$ day, carbamazepine $800 \mathrm{mg} /$ day, lamotrigine $100 \mathrm{mg} /$ day), his seizures continued at a frequency of one or two per month.

A cranial MRI revealed left hippocampal atrophy on the $\mathrm{T}_{1}$-weighted image with increased signal intensity on the $\mathrm{T}_{2}$-weighted image, which was consistent with hippocampal sclerosis (fig. 1a), and ${ }^{18}$ FDG-PET showed mild hypometabolism in the left medial temporal cortex (fig. 1b).
Long-term video-scalp EEG monitoring showed 10 stereotyped seizures manifested by aura and motionless staring, followed by bilateral hand automatisms and repetitive spitting on the bed. Average seizure duration was $31.6 \pm 8.6 \mathrm{~s}$ and average time of propagation to the right hemisphere was $18.8 \pm 5.0 \mathrm{~s}$. Ictal spitting usually occurred $19.5 \pm 5.2 \mathrm{~s}$ after clinical seizure onset. Ictal scalp EEG with sphenoidal electrodes was of no value for either lateralization or localization. There were no identifiable interictal epileptiform discharges on scalp EEG with sphenoidal electrodes. An ictal SPECT showed mild hyperperfusion in the left temporal lobe and right thalamus. IAP demonstrated language dominance for the left hemisphere. Intracranial monitoring was performed using bilateral mesial temporal depth ( 1 on each side, 8 contact points per electrode) and mesial and lateral temporal subdural strip electrodes (2 on each side, 4 contact points per electrode) to determine the precise onset area of his epileptic seizures. During intracranial EEG monitoring, a total of 10 electrographic seizures were recorded. Of these 10 seizures, 9 seizures revealed that fast (15$20 \mathrm{~Hz}$ ) ictal rhythms were recruited in the left hippocampal depth electrodes and propagated to the right hippocampus depth electrodes after which spitting automatisms were evident (fig. 2a). Ictal

\section{KARGER}

Fax +41 613061234 E-Mail karger@karger.ch www.karger.com www.karger.com/ene
Joong Koo Kang, MD, PhD

Department of Neurology, University of Ulsan College of Medicine

Asan Medical Center, 388-1, Pungnap-dong, Songpa-gu

Seoul 138-736 (South Korea)

Tel. +82 23010 3448, Fax +82 2474 4691, E-Mail jkkang@amc.seoul.kr 
Fig. 1. Neuroimaging findings. a $\mathrm{T}_{2}$-weighted MR image shows left hippocampal atrophy with hyperintense $\mathrm{T}_{2}$ signal (arrowhead). b ${ }^{18}$ FDG-PET shows left medial temporal hypometabolism (arrow).
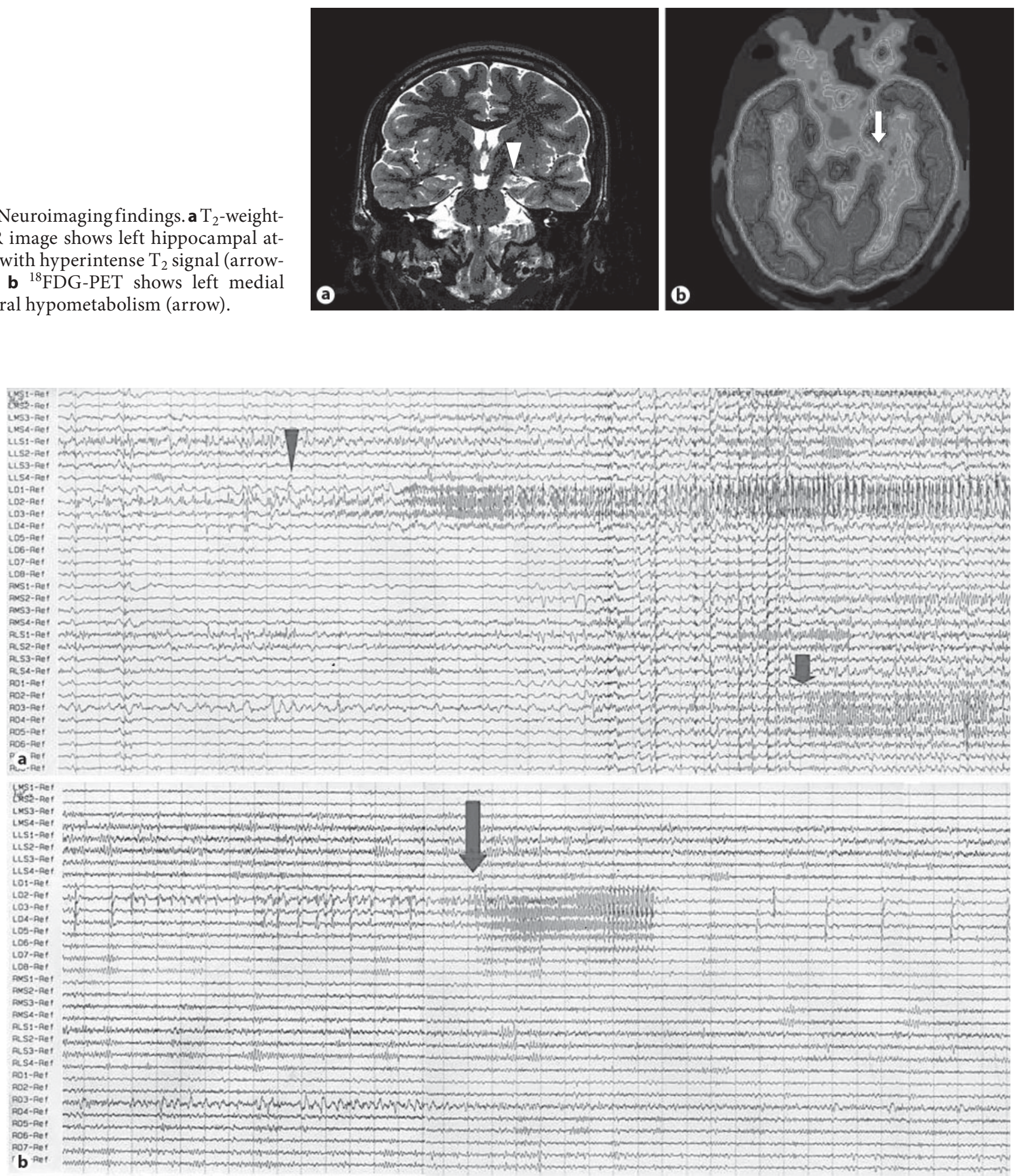

Fig. 2. Intracranial EEG findings. a The seizure starts with rhythmic activity in the left depth electrodes (LD1-LD4) contacting amygdala and hippocampus (arrowhead). The patient feels aura several seconds after seizure propagation to the right medial temporal lobe (short arrow). The seizure activity propagates with fast rhythmic $(10-15 \mathrm{~Hz})$ recruiting in the right depth electrodes contacting amygdala and hippocampus (RD1-RD4), after which spitting automatism emerged. b One of the 10 seizures originated at the left mesial temporal lobe, but was confined to the left hemisphere, and was manifested as aura and motionless staring without spitting automatism (long arrow). 
spitting occurred $21.5 \pm 5 \mathrm{~s}$ after EEG onset and EEG seizure durations were usually $31.1 \pm 8.6 \mathrm{~s}$. One of the 10 seizures originated at the left mesial temporal lobe, but was confined to the left hemisphere, and was manifested as aura and motionless staring without spitting automatism (fig. 2b). Intracranial EEG and semiological analyses led to a conclusion that the epileptogenic area was the left mesial temporal lobe and the symptomatogenic area for spitting automatism was the right hemisphere. Left anterior temporal lobectomy with amygdalohippocampectomy was performed, and at the time of writing this patient had remained seizure-free without antiepileptic medication for 2 years after surgery.

\section{Discussion}

Ictal spitting is a rare epileptic event and only occurs in approximately $0.3 \%$ of the monitored epilepsy population [4]. In a study on the differentiating clinical features of right and left temporal lobe seizures, ictal spitting was exclusively observed in patients with right temporal lobe seizures [5]. Voss et al. [6] reviewed 2,500 epilepsy patients and found only 5 ictal spitting cases with a lesion in the right temporal lobe. Moreover, in all patients, resection of the right temporal lobe produced a seizure-free state. However, patients with ictal spitting originating from the left temporal lobe have been reported even more rarely.

Ozkara et al. [7] reported 1 patient with seizures originating in the left hemisphere, in whom the right hemisphere was dominant for language. Other patients with seizures arising from the left hemisphere have been described, but no mention was made of language lateralization $[8,9]$.

Kellinghaus et al. [4] described 12 patients with ictal spitting, including 1 patient in whom seizures were determined to have been caused by dominant TLE by IAP and invasive EEG. However, they performed a unilateral invasive study in the left side only. In view of the possible discrepancy between epileptogenic and symptomatogenic areas for spitting automatism, such as in our case, unilateral invasive monitoring is insufficient for identification of the anatomical structure of spitting automatism whether it originated from the dominant hemisphere even though the epileptogenic area was the dominant temporal lobe.

In our case, surface EEG was not helpful in localizing or lateralizing the epileptogenic area, and the epileptogenic area was eventually localized to the left mesial temporal lobe by invasive study using bilateral depth and strip electrodes. However, the symptomatogenic area for ictal spitting turned out to be the right hemisphere, because spitting automatism occurred after ictal rhythms propagated to the right temporal depth electrodes. One seizure without spitting automatism was recorded in our patient, during which the ictal rhythm originated from the left mesial temporal lobe as previous seizures and terminated without propagating to the right hemisphere. Moreover, the corresponding sequence of semiology was identical to the previous nine seizure events, except for the ictal spitting. These findings suggest that ictal spitting arose in our patient due to right hemisphere involvement, though the seizures originated in the dominant hemisphere. Also the localization for ictal spitting may have been the right temporal lobe in this patient, because it occurred immediately after ictal rhythm had propagated to the right temporal depth electrodes. However, we could not conclude on the precise anatomical structure of ictal spitting, because the present study was conducted with limited numbers of intracranial electrodes that monitored only the bilateral mesial temporal and lateral temporal lobes.

As described above, all previously published cases with ictal spitting originating from the dominant hemisphere have not had enough evidence for identification of the anatomic structure of spitting automatism. Thus, the present case is of value because it documents the symptomatogenic area for ictal spitting, i.e. the nondominant hemisphere, using bilateral depth and strip electrodes, even in a case of dominant temporal lobe seizure.

To our knowledge, this is the first case documented by a bilateral invasive study of ictal spitting originating from dominant TLE. Moreover, this case suggests an important notion, namely, that the symptomatogenic area responsible for spitting automatism may be the nondominant hemisphere, even when the epileptogenic area is located in the dominant hemisphere.

\section{References}

1 Benarroch EE: The central autonomic network: functional organization, dysfunction, and perspective. Mayo Clin Proc 1993;68: 988-1001.

2 Kotagal P, Luders HO, Williams G, Nichols TR, McPherson J: Psychomotor seizures of temporal lobe onset: analysis of symptom clusters and sequences. Epilepsy Res 1995; 20:49-67.

3 Devinsky O, Frasca J, Pacia SV, Luciano DJ, Paraiso J, Doyle W: Ictus emeticus: further evidence of nondominant temporal involvement. Neurology 1995;45:1158-1160.

4 Kellinghaus C, Loddenkemper T, Kotagal P: Ictal spitting: clinical and electroencephalographic features. Epilepsia 2003;44:10641069.

5 Dantas FG, Yacubian EM, Jorge CL, Pedreira CC, Bueno JF, Valerio RM: Clinical and EEG analysis of mesial and lateral temporal lobe seizure. Arq Neuropsiquiatr 1998;56:341349.

6 Voss NF, Davies KG, Boop FA, Montouris GD, Hermann BP: Spitting automatism in complex partial seizures: a nondominant temporal localizing sign. Epilepsia 1999;40: 114-116.

7 Ozkara C, Hanoglu L, Eskazan E, Kulaksizogvlu IB, Ozyurt E: Ictal spitting during a left temporal lobe originated complex partial seizure: a case report. Cigdem Epileptic Disord 2000;2:169-171.

8 Angelika H, Frederick A, Ernst A: Spitting automatism in temporal lobe seizures with a brief review of ethological and phylogenetic aspects of spitting. Epilepsia 1972;13:767772 .

9 Carmant L, Rivielo JJ, Thiele EA: Compulsory spitting: an unusual manifestation of temporal lobe epilepsy. Epilepsy 1994;7:167170. 